\title{
Quasi-bound low energy tail of resonance
}

\author{
I. Stefan, F. de Oliveira Santos, M.G. Pellegriti, M. Angélique, J.C.
} Dalouzy, F. de Grancey, M. Fadil, S. Grévy, M. Lenhardt, M. Lewitowicz, A. Navin, L. Perrot, M.G. Saint Laurent, I. Ray, O. Sorlin, C. Stodel, J.C. Thomas*, G. Dumitru, A. Buta, R. Borcea, F. Negoita, D. Pantelica , J.C. Angélique**, E. Berthoumieux $^{*}$, A. Coc, J. Kiener, A. Lefebvre-Schuhl, V.

Tatischeff $^{\S}$, J.M. Daugas, O. Roig ${ }^{\mathbb{I I}}$, T. Davinson ${ }^{\|}$and M. Stanoiu ${ }^{\dagger \dagger}$

\author{
${ }^{*}$ Grand Accélérateur National d'Ions Lourds UMR 6415 B.P. 5027 F-14076 Caen Cedex, France \\ ${ }^{\dagger}$ Horia Hulubei National Institute of Physics and Nuclear Engineering P.O. Box MG6 \\ Bucharest-Margurele, Romania \\ ${ }^{* *}$ Laboratoire de Physique Corpusculaire CNRS-IN2P3 UMR 6534 ISMRA et Université de Caen \\ F-14050 Caen, France \\ ${ }^{\ddagger}$ CEA Saclay DSM/DAPNIA/SPHN F-91191 Gif-sur-Yvette, France \\ ${ }^{\S}$ CSNSM UMR 8609 CNRS-IN2P3/Univ.Paris-Sud Bât. 10491405 Orsay Campus, France

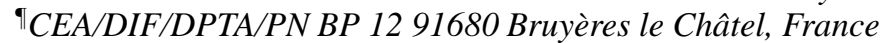 \\ "Department of Physics and Astronomy University of Edinburgh Edinburgh EH9 3JZ, United \\ Kingdom \\ ${ }^{\dagger}$ Institut de Physique Nucléaire UMR 8608 CNRS-IN2P3/Univ.Paris-Sud F-91406 Orsay, France
}

\begin{abstract}
The ground state of particle-unbound nuclei is observed as a resonance. According to the Heisenberg's uncertainty principle, the shorter is the lifetime of the state the broader is the resonance. The Breit-Wigner formalism describes perfectly the shape of the resonance when energydependent partial-widths are used. In the low energy tail of a ground state resonance near the particle-emission threshold, the partial-width for the emission of a charged particle through the Coulomb barrier is drastically reduced. The fact that the proton decay is strongly hindered seems to be equivalent to the assertion that the decay of the proton is strongly delayed. In this interpretation and as an example, possible consequences are discussed in astrophysical context. Two reactions ${ }^{15} \mathrm{O}\left(\mathrm{p}, \beta^{+}\right){ }^{16} \mathrm{O}$ and ${ }^{15} \mathrm{O}(\mathrm{p}, \gamma)\left(\beta^{+}\right){ }^{16} \mathrm{O}$ are proposed as new pathways to bypass the ${ }^{15} \mathrm{O}$ waiting point. Cross-sections were calculated after precise measurements of energies and widths of the protonunbound ${ }^{16} \mathrm{~F}$ low lying states, obtained using the $\mathrm{H}\left({ }^{15} \mathrm{O}, \mathrm{p}\right){ }^{15} \mathrm{O}$ reaction. These measurements were performed at SPIRAL - GANIL facility using an ${ }^{15} \mathrm{O}$ post-accelerated radioactive beam.
\end{abstract}

\section{INTRODUCTION}

The proton-unbound nucleus ${ }^{16} \mathrm{~F}$ probably play an important role in X-ray bursts. These astronomical events are known to happen in close binary systems, where accretion takes place from an extended companion star on the surface of a neutron star (type I X-ray bursts). The accreted matter is compressed until it reaches sufficiently high pressure conditions to trigger a thermonuclear runaway. In these explosive events, ${ }^{14} \mathrm{~N}$ nuclei are mainly transformed into ${ }^{15} \mathrm{O}$ by proton captures [1, 2]. Then, the pathway for new proton captures is hindered by the proton-unbound nucleus ${ }^{16} \mathrm{~F}$. This leads to an equilibrium between formation and decay of this proton-unbound nucleus. The reaction flux and the energy generation are then limited by the relatively slow $\beta^{+}$-decay of ${ }^{15} \mathrm{O}\left(\mathrm{t}_{1 / 2}=122 \mathrm{~s}\right)$, 
which create a waiting point. From time to time before the proton is emitted, ${ }^{16} \mathrm{~F}$ can capture another proton thus producing the ${ }^{17} \mathrm{Ne}$ particle stable isotope. This two-proton capture process was calculated by Goerres et al. [3] and later by Grigorenko in [4], and it was found to be significant for extreme densities (larger than $10^{11} \mathrm{~g} / \mathrm{cm}^{3}$ ). In this study, $\beta^{+}$-decay of ${ }^{16} \mathrm{~F}$ to ${ }^{16} \mathrm{O}$ is proposed as an alternative channel. The calculation of this ${ }^{15} \mathrm{O}\left(p, \beta^{+}\right){ }^{16} \mathrm{O}$ reaction requires the knowledge of the properties of the low lying states in ${ }^{16} \mathrm{~F}$. Here it is reported on an accurate experiment about this nucleus. Alternative reaction channels are discussed hereafter.

\section{SPECTROSCOPY OF ${ }^{16} \mathbf{F}$}

A beam of radioactive ${ }^{15} \mathrm{O}$ nuclei was produced at the SPIRAL-GANIL facility through the projectile fragmentation of a $95 \mathrm{~A} . \mathrm{MeV}{ }^{16} \mathrm{O}$ primary beam on a thick carbon target. Mean intensities of $10^{7}$ pps at an energy of $1.2 \mathrm{~A} . \mathrm{MeV}$ were obtained after post acceleration. A beam contamination of less than $1 \%$ of ${ }^{15} \mathrm{~N}$ was achieved in the analysis line of LISE spectrometer [5] where the measurements were made. Two stable beams, ${ }^{14} \mathrm{~N}$ and ${ }^{15} \mathrm{~N}$, were also used in similar experimental conditions for calibrations. The excitation function for the elastic scattering at these low energies can be described by the Rutherford scattering formula, but shows "anomalies", i.e. various resonances that are related to discrete states in the compound nucleus. The principle of the measurement is described in [6, 7] and references therein. A 31(1) $\mu \mathrm{m}$ thick polyethylene $\left(\mathrm{CH}_{2}\right)_{n}$ target was used, thick enough to stop the beam inside. The scattered protons were detected by a silicon detector, placed at forward angles $\left(180^{\circ}\right.$ in the center of mass frame) within an angular acceptance of $2^{\circ}$. Protons were identified using their energy and time-of-flight. The energy resolution was $4 \mathrm{keV}$ in the center of mass (c.m.) frame. Fig. 1 shows the excitation function for the $\mathrm{H}\left({ }^{15} \mathrm{O}, p\right){ }^{15} \mathrm{O}$ reaction measured from $0.450 \mathrm{MeV}$ to $1.1 \mathrm{MeV}$.

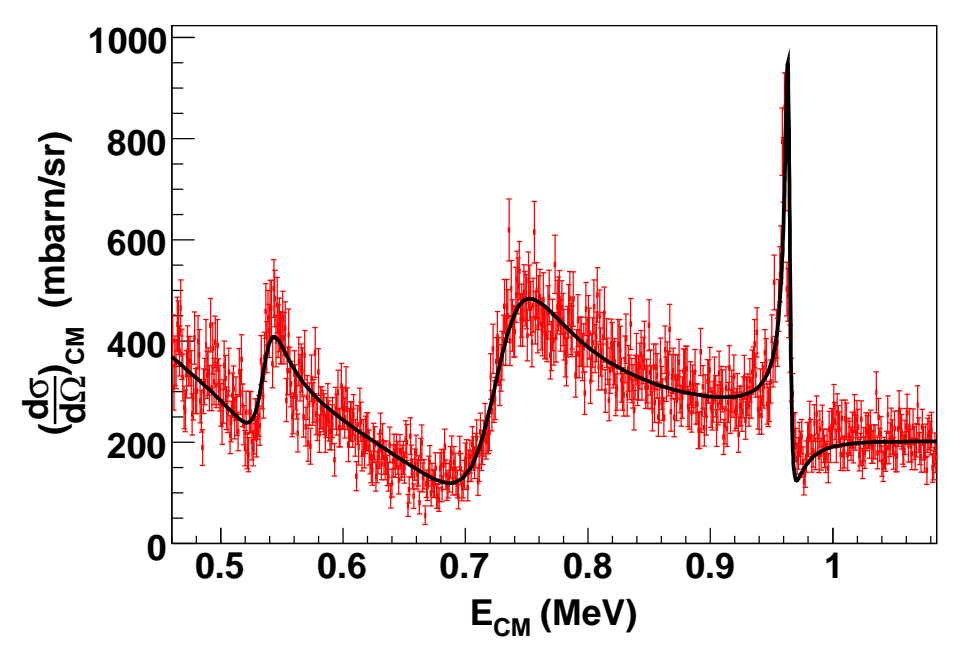

FIGURE 1. Excitation function for the $\mathrm{H}\left({ }^{15} \mathrm{O}, p\right){ }^{15} \mathrm{O}$ reaction at $180^{\circ}$ in c.m.. The line is a result of an R-matrix calculation using parameters from Table I.

The measured cross section was reproduced by an R-matrix [8] calculation using the 
code ANARKI [9] which is seen to be in a good agreement with the data. A value of $S_{p}=-536 \pm 5 \mathrm{keV}$ was obtained for the proton separation energy in agreement with the recommended value [10]. The R-matrix analysis was also used to extract the properties of the first three states in ${ }^{16} \mathrm{~F}$, given in Table I. A significant difference was found between the present and the recommended value of the width for the first excited state [11]. This width is an important parameter used in the calculations presented in the next section.

TABLE 1. Measured properties for the low-lying states in ${ }^{16} \mathrm{~F}$.

\begin{tabular}{|ccc|ccc|}
\hline \multicolumn{2}{|c|}{ Recommended values [1]] } & \multicolumn{3}{|c|}{ This work } \\
$\mathrm{J}^{\pi}$ & $\mathrm{E}_{x}(\mathrm{keV})$ & $\Gamma_{p}(\mathrm{keV})$ & $\mathrm{E}_{C M}(\mathrm{keV})$ & $\mathrm{E}_{x}(\mathrm{keV})$ & $\Gamma_{p}(\mathrm{keV})$ \\
\hline $0^{-}$ & 0 & $40 \pm 20$ & $534 \pm 5$ & 0 & $25 \pm 10$ \\
$1^{-}$ & $193 \pm 6$ & $<40$ & $732 \pm 10$ & $198 \pm 10$ & $70 \pm 5$ \\
$2^{-}$ & $424 \pm 5$ & $40 \pm 30$ & $958 \pm 2$ & $425 \pm 2$ & $6 \pm 3$ \\
\hline
\end{tabular}

\section{CALCULATION OF ${ }^{15} \mathrm{O}\left(P, \beta^{+}\right){ }^{16} \mathrm{O}$ AND ${ }^{15} \mathrm{O}(P, \gamma)\left(\beta^{+}\right){ }^{16} \mathrm{O}$}

Calculation of the ${ }^{15} \mathrm{O}\left(p, \beta^{+}\right){ }^{16} \mathrm{O}$ reaction cross section was performed using the Breit Wigner formalism and properties of ${ }^{16} \mathrm{~F}$ determined in this experiment. The cross section of this reaction was found to be extremely small as it is shown in figure 2, always lower than $10^{-19}$ barns.

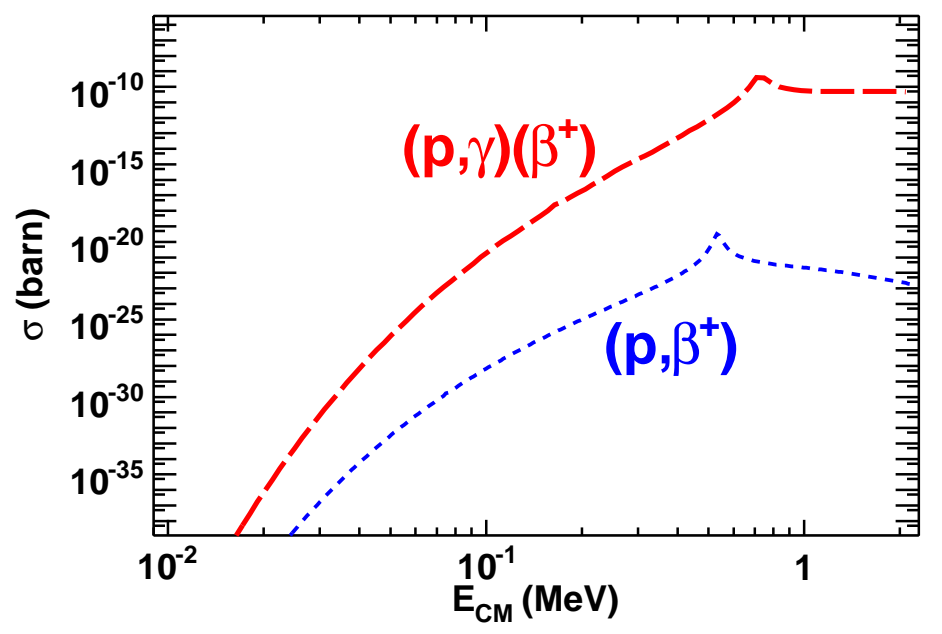

FIGURE 2. Calculated cross sections for the ${ }^{15} \mathrm{O}\left(p, \beta^{+}\right)^{16} \mathrm{O}$ and the ${ }^{15} \mathrm{O}(p, \gamma)\left(\beta^{+}\right){ }^{16} \mathrm{O}$ reaction (see text).

A similar but alternative reaction ${ }^{15} \mathrm{O}(p, \gamma)\left(\beta^{+}\right){ }^{16} \mathrm{O}$ is proposed. The calculation of this reaction was performed sequentially, a schematic representation of this reaction is shown in Fig. 3. Proton capture reaction to the first excited state of ${ }^{16} \mathrm{~F}$ is considered, followed by a $\gamma$ decay to the g.s. resonance, from which a $\beta^{+}$-decay branching ratio is taken into account. 


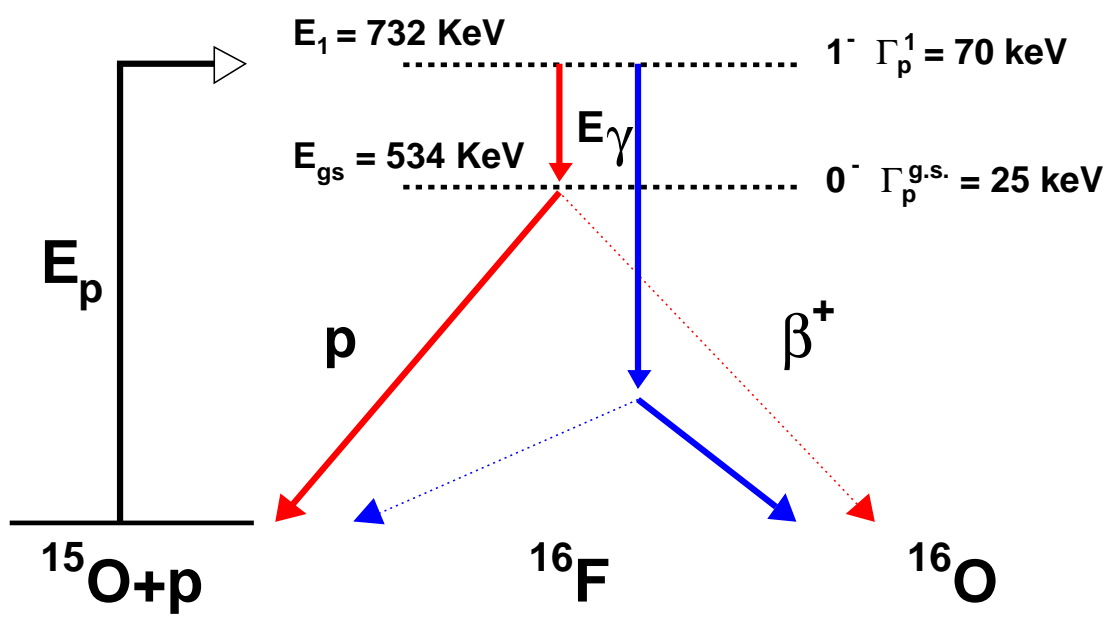

FIGURE 3. Schematic representation of the ${ }^{15} \mathrm{O}(p, \gamma)\left(\beta^{+}\right){ }^{16} \mathrm{O}$ reaction (see text). Two cases are represented. In red, $\gamma$-transitions populate the ${ }^{16} \mathrm{~F}$ g.s. at the resonance energy. In that case, ${ }^{16} \mathrm{~F}$ mainly decays by proton emission. In blue, high energy $\gamma$-transitions populate the low energy wing of the g.s. resonance. In that case, $\beta^{+}$-decay dominates.

It is assumed that the cross section $\sigma_{p \gamma \beta}\left(E_{p}\right)$ for the $(p, \gamma)\left(\beta^{+}\right)$reaction at the energy $\mathrm{E}_{p}$ is an integration of the differential cross section over all possible energies of the $\gamma$ transition (since the g.s. has a large width):

$$
\sigma_{p \gamma \beta}\left(E_{p}\right)=\int \sigma_{p \gamma}\left(E_{p}, E_{\gamma}\right) P_{\gamma}\left(E_{\gamma}\right) P_{\beta}\left(E_{p}, E_{\gamma}\right) d E_{\gamma}
$$

where $\sigma_{p \gamma}\left(E_{p}, E_{\gamma}\right)$ is the cross section to capture the proton at the energy $\mathrm{E}_{p}$ and to emit a $\gamma$-ray with an energy $\mathrm{E}_{\gamma}, P_{\gamma}\left(E_{\gamma}\right) d E_{\gamma}$ is the strength function, that is the probability for the $\gamma$-ray to have an energy between $E_{\gamma}$ and $E_{\gamma}+d E_{\gamma}$, and $P_{\beta}\left(E_{p}, E_{\gamma}\right)$ is the branching ratio function for the ${ }^{16} \mathrm{~F}$ nucleus to decay by $\beta^{+}$-ray emission. The different terms of this formula are detailed in [12]. Naively, one might have expected to obtain a small cross section for the $(p, \gamma)\left(\beta^{+}\right)$reaction, similar to the $\left(p, \beta^{+}\right)$one, since $\gamma$ - and $\beta$-widths are much smaller than proton-widths. Contrary to naive expectations, the $(p, \gamma)\left(\beta^{+}\right)$cross section is about $10^{10}$ times larger than the $\left(p, \beta^{+}\right)$cross section, as shown in Fig. 2. The large ratio can be explained in the following way. There is only one $\left(p, \beta^{+}\right)$reaction for $10^{20}(p, p)$ reactions (at the resonance energy). In the $(p, \gamma)\left(\beta^{+}\right)$case, one $\gamma$-ray is emitted for $10^{8}(p, p)$ reactions (from the ratio of the widths) and about one $\gamma$-transition over $10^{3}$ populates the low energy wing of the g.s. resonance (less than $15 \mathrm{keV}$ above the proton-emission threshold) where it is almost always followed by a $\beta^{+}$-decay $\left(\mathrm{P}_{\beta} \simeq 1\right)$. This implies that one captured proton over $10^{11}(\mathrm{p}, \mathrm{p})$ reactions induces a $(p, \gamma)\left(\beta^{+}\right)$ reaction, that is a factor $10^{9}$ times larger than in the $\left(p, \beta^{+}\right)$reaction. This is the main explanation of the large factor between the $(p, \gamma)\left(\beta^{+}\right)$and the $\left(p, \beta^{+}\right)$reaction cross sections. 


\section{DISCUSSION}

Particle-unbound states in nuclei are observed as resonances. According to the Heisenberg's uncertainty principle, the shorter is the lifetime of the state the broader is the resonance. The Breit-Wigner formalism describes perfectly the shape of the resonance when the energy dependence of the partial-widths is taken into account. This formalism is commonly used in astrophysics to calculate cross sections of resonant reactions at very low energies. In light unbound nuclei in the proton-rich side of the nuclear chart, in ${ }^{16} \mathrm{~F}$ for example, even the ground state is unbound and it is observed as a broad resonance. In the low energy tail of the ground state resonance near the proton-emission threshold, the partial-width for the emission of the proton through the Coulomb barrier is drastically reduced. This is due to the fact that the proton has to tunnel through the large Coulomb potential barrier of the core, ${ }^{15} \mathrm{O}$ nucleus in the previous example. The fact that the proton decay is strongly hindered seems to be equivalent to the assertion that the decay of the proton is strongly delayed. In other words, it seems that the proton is trapped inside the nucleus. To our knowledge this crucial idea has never been discussed. In astrophysics, this assertion is implicitly used in the calculation of the reactions rates involving unbound nuclei. The triple alpha reaction involves the unbound ${ }^{8} \mathrm{Be}$ nucleus. In the study of Nomoto et al. [13], the formalism used to calculate the rate of the reaction use implicitly this assertion, and in the NACRE compilation of reaction rates [14] it is written about this calculation "...the rate assumes the ${ }^{8} \mathrm{Be}$ ground state has been formed at an energy E different from resonance energy, and that it is bound".

In this study, two reactions channels ${ }^{15} \mathrm{O}\left(p, \beta^{+}\right){ }^{16} \mathrm{O}$ and ${ }^{15} \mathrm{O}(p, \gamma)\left(\beta^{+}\right){ }^{16} \mathrm{O}$ are studied. Both reactions eventually proceed through the $\beta^{+}$-decay of the intermediate unbound ${ }^{16} \mathrm{~F}$ g.s., which is fed directly by a proton capture or indirectly through a proton capture to the first excited state followed by a $\gamma$-emission. To calculate the cross section of these reactions, it is used the assertion that the low energy tail of ${ }^{16} \mathrm{~F}$ is quasi-bound. In the following, several aspects of this idea are discussed:

1. Validity of equ. (1). The validity of equ. (1) to calculate the $(p, \gamma)\left(\beta^{+}\right)$cross section is questionable. This equation is equivalent to the assertion that the $(p, \gamma)\left(\beta^{+}\right)$ reaction is a pure sequential process. The triple alpha reaction is a similar kind of reaction, that involves an intermediate unbound nucleus $\left({ }^{8} \mathrm{Be}\right)$. Implicitly, the calculation of this reaction is performed using the same assertion as used in our study, that is the alpha are captured sequentially [15]. The effect of the assumption made in equ. (1) could be evaluated by using a three-body formalism, as proposed for example by L. V. Grigorenko [ $₫$ ] to calculate the ${ }^{15} \mathrm{O}(2 p, \gamma){ }^{17} \mathrm{Ne}$ reaction.

2. Validity of the strength function. The strength function that describes the ${ }^{16} \mathrm{~F}$ ground state resonance when populated by gamma transitions is not known, here it is used a standard Breit-Wigner shape with energy-dependent widths. The validity of this description remains to be confirmed theoretically and experimentally. In any case, the feeding of the low energy tail of the ${ }^{16} \mathrm{~F}$ ground state resonance by $\gamma$-transitions will remain larger than its feeding by direct proton captures.

3. Will ${ }^{16}$ F survive a long time ? In quantum mechanics, decay probability and lifetime are equivalent quantities. Because time-reversal invariance holds for electromagnetic and nuclear forces, the partial width is independent of the direction of the 
process. The partial width for the formation of the compound nucleus ${ }^{16} \mathrm{~F}$ through an entrance channel ${ }^{15} \mathrm{O}+\mathrm{p}$ at an energy $\mathrm{E}$ is identical with the partial width for the decay of the ${ }^{16} \mathrm{~F}$ nucleus produced at the same energy. The partial width for the formation of ${ }^{16} \mathrm{~F}$ being reduced at low energy by the Coulomb barrier, the proton decay width is reduced as well. Implicitly, a Breit-Wigner shape with no energy dependance for the widths and with no threshold limits means that the state decays according to an exponential law [16]. Here, the energy dependence of the widths is used, the state is broad and it is located close to the proton emission threshold, these are conditions that strongly restrict the validity of the exponential law. The change of the decay law would cause a delayed proton emission. The study of this peculiar aspect is strongly required. Does that mean ${ }^{16} \mathrm{~F}$ will survive a long time when populated in the low energy tail of the ground state? A time long enough that the beta-decay can compete with the proton emission ? This remains to be observed experimentally.

\section{REFERENCES}

1. R. Wallace, and S. Woosley, Astrophys. J. Suppl. Ser. 45, 389 (1981).

2. M. Wiescher, H. Schatz, and A. Champagne, Phil. Trans. Roy. Soc. London A 356, 2105 (1998).

3. J. Görres et al., Phys. Rev. C 51, 392 (1995).

4. L. V. Grigorenko et al., Phys. Rev. C 72, 015803 (2005).

5. R. Anne et al., Nucl. Instr. and Meth. A 257, 215 (1987).

6. V. Z. Golberg et al., Phys. At. Nucl. 60, 1061 (1997).

7. F. de Oliveira Santos et al., Eur. Phys. J. A 24, 237 (2005).

8. A. Lane, and R. Thomas, Rev. Mod. Phys. 30, 257 (1958).

9. E. Berthoumieux et al., Nucl. Instr. and Meth. B 55, 136-138 (1998).

10. G. Audi et al., Nucl. Phys. A 729, 337 (2003).

11. D. Tilley et al., Nucl. Phys. A 564, 1 (1993).

12. I. Stefan et al., Tours Symposium On Nuclear Physics VI AIP Conference Proceedings 891, 155 (2007).

13. K. Nomoto, F.-K. Thielemann, and S. Miyaji, Astron. Astrophys. 149, 239 (1985).

14. C. Angulo et al., Nucl. Phys. A 656, 3-187 (1999).

15. K. Langanke et al., Z. Phys. A 324, 147 (1986).

16. N. G. Kelkar et al., Phys. Rev. C 70, 024601 (2004). 